\title{
Free-Volume Formation and Relaxation in a Poly(ethylene oxide)/ Poly(methyl methacrylate) Blend I. Detection by the Spin Label Method
}

\author{
Shigetaka SHIMADA \\ Nagoya Institute of Technology, Gokiso-cho, Showa-ku, Nagoya 466, Japan
}

(Received October 25, 1995)

\begin{abstract}
The electron spin resonance (ESR) line shape of nitroxide radical labels attached to poly(ethylene oxide) (PEO) chains in a blend with isotactic poly(methyl methacrylate) (PMMA) was studied. Two spectral components with different rates of motion, a "fast" component and a "slow" component arising from the radicals, are observed. The ESR spectra observed at room temperature change substantially as a function of annealing and aging temperatures. The fractional amount of the fast component (mobile fraction) was estimated from the ESR spectra. (I) When the blend is annealed at temperature above $23^{\circ} \mathrm{C}$, the mobile fraction increases in the lower temperature range and decreases in the higher temperature range. These phenomena can be attributed to phase separation and molecular mixing. (II) When the blend is phase-separated and subsequently aged at temperatures below $23^{\circ} \mathrm{C}$, surprisingly the mobile fraction decreases with decreasing aging temperature around $-13^{\circ} \mathrm{C}$. When the sample is again annealed above $23^{\circ} \mathrm{C}$, the mobile fraction increases abruptly at $60^{\circ} \mathrm{C}$. These phenomena can be attributed to free-volume relaxation and formation. Relaxation phenomena should come from different rates of molecular motion of PMMA and PEO chains in the phase. The ESR spectra of nitroxide labels attached to PMMA chains in the blend support the conclusions.

KEY WORDS Electron Spin Resonance / Spin Label / Polymer Blend / Phase Separation / Molecular Mixing / Molecular Motion / Free Volume / Relaxation Phenomena /
\end{abstract}

Physical aging or annealing of a polymer blend gives rise to progressive change in its properties. For example, when the polymer blend has an upper critical solution temperature, molecular mixing proceeds on annealing above the critical temperature, and phase separation occurs on storing below the temperature. ${ }^{1}$ Volume $^{2}$ and enthalpy relaxation ${ }^{3}$ also occurs in the glassy state of the blend, having its origin in non equilibrium character of this state and consequent relaxation toward equilibrium.

This study reports an electron spin resonance (ESR) method for the determination of the distribution of free-volume sites in a poly(ethylene oxide) (PEO)/poly(methyl methacrylate) (PMMA) blend. Some authors have studied blends of PEO and PMMA. ${ }^{4}$ One of the conclusions is that one homogeneous amorphous phase is present for PEO/atactic PMMA and PEO/syndiotactic PMMA blends, whereas the PEO/isotactic PMMA amorphous system is phase separated. We have been interested in molecular structures and molecular mobility in such a complicated system.

We developed spin-label techniques to study the structure and dynamic behavior of polymer chains at a particular site or in a particular region. ${ }^{5}$ In our previous paper, ${ }^{6}$ ESR spectra of the nitroxide label reflected different environments in a PEO/PMMA blend, with different rates of motion, a "fast" component and a "slow" component, referring to the appearance of isotropic spectra due to fast motion and of anisotropic spectra for immobilized labels. Temperature dependence of the composite ESR spectra indicates a heterogeneous medium and therefore phase separation or concentration fluctuation in the polymer blend. The temperature change of the ESR spectra are reversible with slowly increasing and decreasing temperature. We also studied the freevolume relaxation around $-13^{\circ} \mathrm{C}$ in the blend by the PEO spin-label method.
In general, the states of aggregation of a polymer substance at an annealing temperature are frozen at room temperature by rapid quenching in comparison with molecular mobility. In this study, we present detailed experimental results of changes of the composite ESR spectra observed at room temperature with annealing and aging temperatures and propose a simple model mechanism for free-volume formation and relaxation in the blend.

\section{EXPERIMENTAL}

\section{Materials}

Spin-labeled PEO(SLPEO) was prepared by the method of Törmälä et al. ${ }^{7}$ 3-Carbonyl-2,2,5,5-tetramethyl pyrroline-1-oxyl radical (Aldrich Chemical Co., Ltd.) was esterified with poly(ethylene oxide) (Katayama Science Co., Ltd.). Isotactic PMMA was prepared by anionic polymerization MMA monomer (Tokyo Kasei Co., Ltd.) using tert-butylmagnesium bromide as a catalyst at $-78^{\circ} \mathrm{C}^{8}$ The number average molecular weight $\left(\bar{M}_{n}\right)$ is $6 \times 10^{3}$ for PEO and $5.0 \times 10^{3}$ for PMMA. Spin-labeled PMMA (SLPMMA) ${ }^{9}$ was prepared to compare mobility with that of SLPEO. After anionic polymerization of methyl methacrylate monomer, nitroxide radical labels were attached to PMMA chain ends by adding spin-label monomer (4-methacryloyloxy)2,2.6,6-tetra-methylpiperidine-1-oxyl). Polydispersity of the SLPMMA is lower than 1.2. Molecular weight and polydispersity were characterized by gel permutation chromatography (GPC), GMX-G1000-G2000-G4000 type apparatus (Tosoh Co., Ltd.).

\section{Preparation of Blends}

Blends were made by mixing SLPEO and PMMA at ratios of $1: 1,1: 4$, and $1: 10$ by weight. Films were 
prepared by casting from $\sim 3 \mathrm{wt} \%$ of benzene solution at $60^{\circ} \mathrm{C}$. The notations used for these samples are EM1, EM4, and EM10. Another blend was also made by mixing PEO, SLPMMA, and PMMA at ratio of $10: 1: 9$ ((PEO)/ (SLPMMA + PMMA) $(1: 1))$ by weight. SLPMMA was diluted to avoid exchange broadening for the ESR spectrum. The notation for the cast film prepared by the same method mentioned above is EMM1.

\section{ESR Measurements}

Samples were stacked in an ESR sample tube and evacuated to $10^{-5} \mathrm{mmHg}$. ESR measurements were carried out with a JEOL FE3XG spectrometer with a PC9801 computer. The magnetic field sweep was calibrated with the known splitting constant of $\mathrm{Mn}^{2+}$.

\section{RESULTS AND DISCUSSION}

\section{Molecular Mixing and Phase Separation}

Figure 1 shows the ESR spectra of SLPEO in the EM 10 blend. The blend sample prepared was annealed at $78^{\circ} \mathrm{C}$ for $30 \mathrm{~min}$ and stored at ambient temperature for 9 days, and then the spectrum (Figure 1a) was observed at room temperature. After the EM10 blend was annealed at $142^{\circ} \mathrm{C}$ for $15 \mathrm{~min}$ and quenched to room temperature, the ESR spectrum (Figure 1b) was observed at room temperature.

In general, the outermost splitting width of the main triplet spectra due to hyperfine coupling caused by the nitrogen nucleus narrows with increasing mobility of radicals because of motional averaging of the anisotropic interactions between electron and nuclear spins. The complete averaging gives rise to the isotropic and narrowed spectrum. The outermost splitting is a good measure of the mobility of the spin-labeled PEO. A two-component spectrum (Figure 1a) in the blend with different rates of motion, a "fast" and a "slow" com- ponent arising from the radicals, is observed when the sample is stored at ambient temperature for a long time. The fast and slow components can be attributed to radicals in the mobile and rigid regions, respectively. Molecular mobility of the PEO chains in the PEO matrices is higher than in the PMMA matrices since the glass-rubber transition temperature $\left(T_{\mathrm{g}}\right)$ of $\mathrm{PEO}\left(-65^{\circ} \mathrm{C}\right)$ is lower than that of isotactic PMMA $\left(60^{\circ} \mathrm{C}\right) .^{4(\mathrm{~b})}$ As discussed previously, ${ }^{6}$ this means that nitroxide radicals are affected by the environment; for example, the states of aggregation, and the fast and slow component spectra (the isotropic and anisotropic spectra) are attributed to SLPEO trapped in the PEO-rich and PMMA-rich regions of the blend, respectively. We can decompose the experimental spectrum into two components and estimate the fractional amount of the fast component (mobile fraction) as mentioned in our previous paper. ${ }^{10}$

The two component spectrum (Figure 1a) converts to only a one component spectrum (Figure $1 \mathrm{~b}$ ) by annealing at the higher temperature. This indicates that SLPEO is molecularly and uniformly mixed with PMMA and the molecular motion of PEO chains is hindered by PMMA chains in a molecularly mixed system. To confirm molecular mixing and phase separation behavior, an elaborate experiment of annealing was made. After observing the spectrum of Figure $1 b$, the sample was annealed at a desired temperature for $30 \mathrm{~min}$, quenched to room temperature and ESR measurements were carried out at the same temperature. The procedure was repeated 10 times with increasing annealing temperature. Figure 2 shows 5 examples of ESR spectra of the SLPEO in the EM10 blend and variation with annealing temperature at 30 to $76^{\circ} \mathrm{C}$. One main change is reduction of the outermost peaks of the slow component and growth of a narrow triplet of the fast component below $40^{\circ} \mathrm{C}$. On the other hand, the intensity of the fast component spectrum decreases above $60^{\circ} \mathrm{C}$. In Figure 3

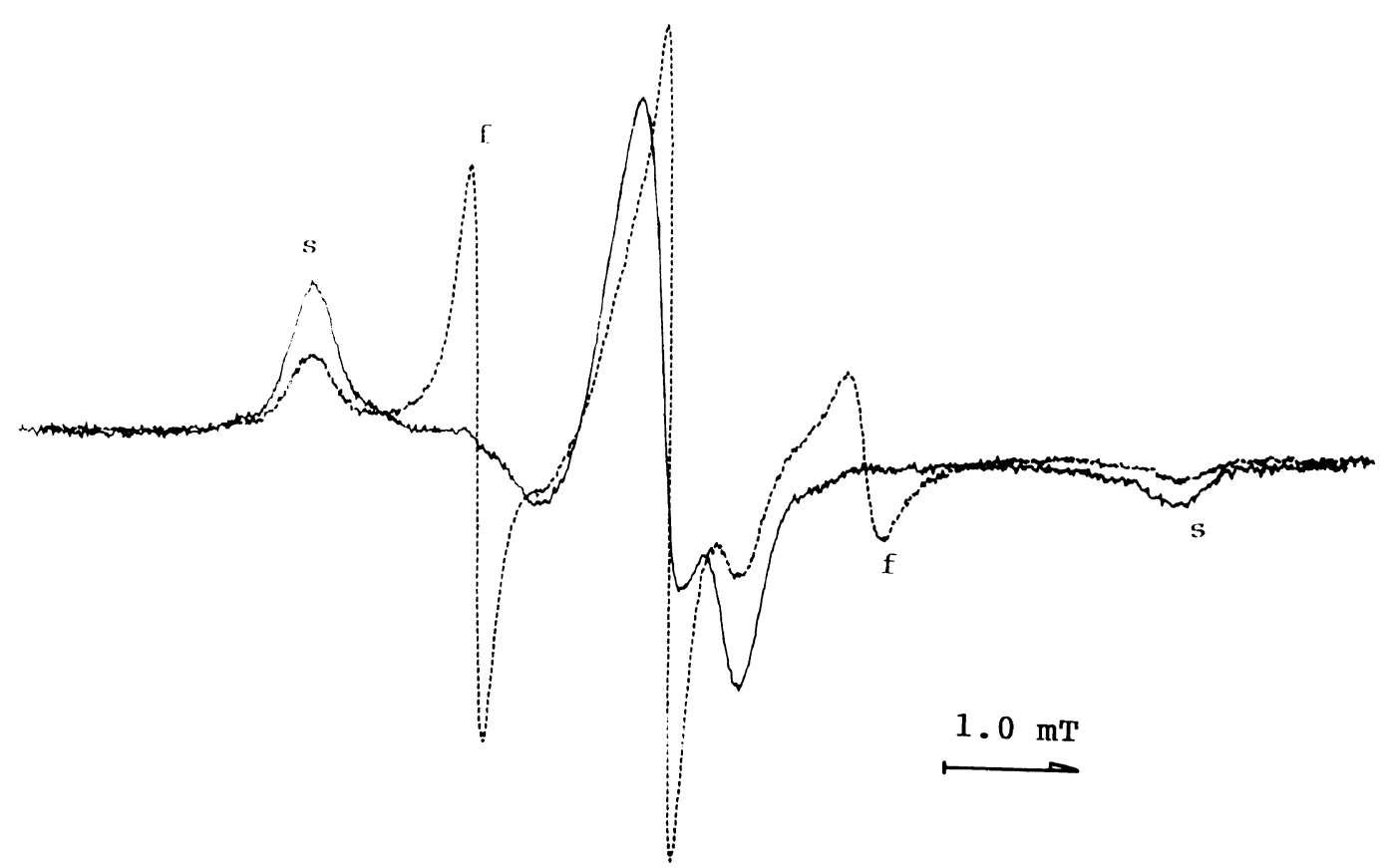

Figure 1. ESR spectra of spin-labeled PEO in PEO/PMMA blend (EM10) after the sample was stored at ambient temperature for 9 days ((a), dotted line) and after the sample was annealed at $142^{\circ} \mathrm{C}$ for $15 \mathrm{~min}((\mathrm{~b})$, solid line). Slow and fast components are indicated by "s" and "f", respectively. Measurements were carried out at $23^{\circ} \mathrm{C}$. (a) ---, after phase separation ( 9 days at room temperature); (b) - after molecular mixing $\left(15 \mathrm{~min}\right.$ at $\left.142^{\circ} \mathrm{C}\right)$. 


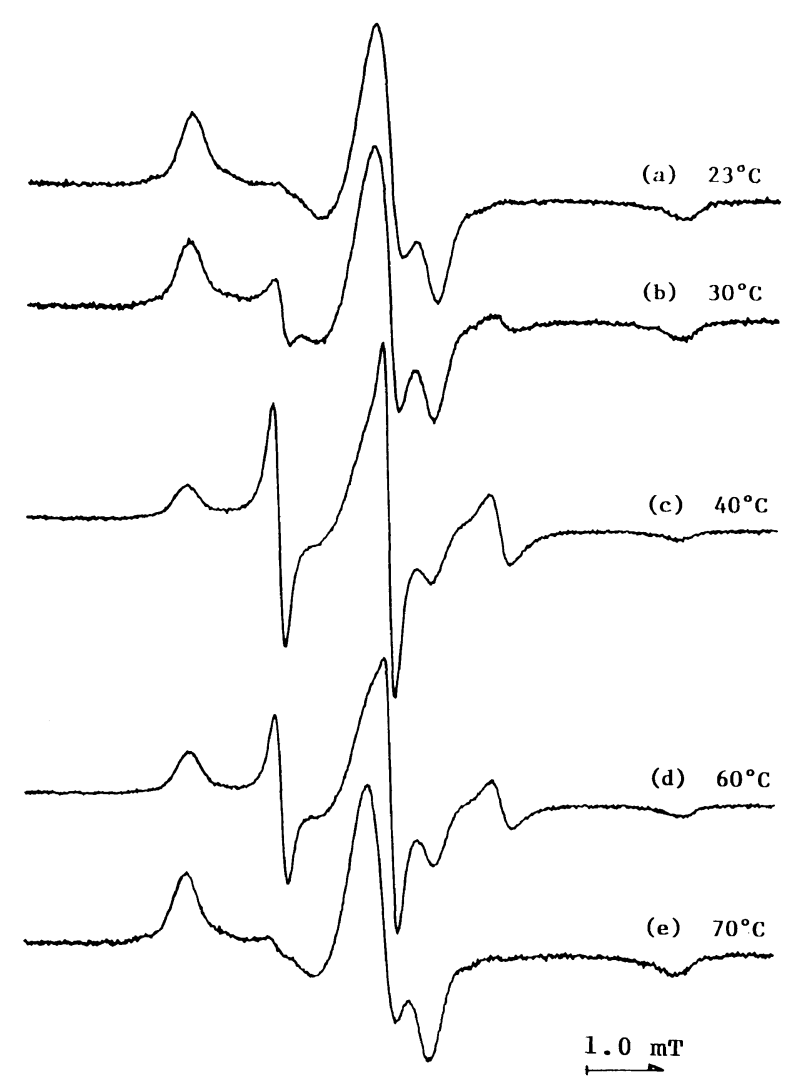

Figure 2. Variation of ESR spectra of spin-labeled PEO in PEO/PMMA blend (EM10) after molecular mixing at annealing temperature. The experiments were carried out after the sample was annealed at $142^{\circ} \mathrm{C}$ for $15 \mathrm{~min}$. (See the text.) ESR spectra were observed at $23^{\circ} \mathrm{C}$.

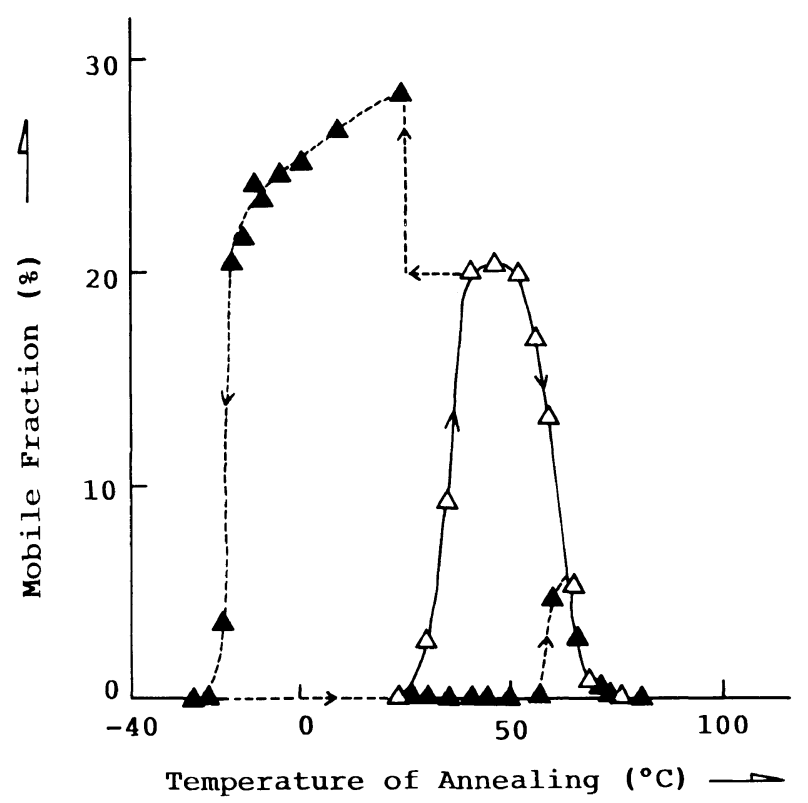

Figure 3. Changes in mobile fraction of ESR spectra of spin-labeled PEO in PEO/PMMA (EM10) with annealing temperature after molecular mixing $(\triangle)$ and with aging temperatures after phase separation $(\mathbf{A})$. The arrows indicate the routes on which changes of the mobile fraction were observed. Measurements were carried out at $23^{\circ} \mathrm{C}$. SLPEO/PMMA $(1: 10):-\triangle-$, after molecular mixing; $-\boldsymbol{\Delta}_{--}$, after phase separation.

$(\triangle)$, the fractional amount of the fast component (mobile fraction) is plotted against annealing temperature. The mobile fraction could be estimated from the relative intensity of the outermost peaks of the slow component spectrum to that in only the one slow component spectrum as shown in Figure $1 \mathrm{~b}$ because integral spectrum intensity was unchanged during the annealing. The mobile fraction has a maximum value around $48^{\circ} \mathrm{C}$ and the fast component spectrum disappears around $68^{\circ} \mathrm{C}$ which coincides with the phase transition temperature found previously. ${ }^{6}$ These facts indicate that phase separation occurs in the lower temperature range, and molecular mixing proceeds in the higher temperature range. Quenching from the molecular mixed state at higher temperatures gives rise to non equilibrium character and phase separation occurs toward equilibrium in the lower temperature range below $48^{\circ} \mathrm{C}$. On the other hand, molecular mixing proceeds with increasing temperature and PEO concentration in the PEO-rich and PMMA-rich phases approaches to a value in the higher temperature range above $48^{\circ} \mathrm{C}$ for upper critical solution temperature (UCST) type behavior.

The same experimental results were obtained for the EM1 sample as shown in Figure $4(O)$. The mobile fraction has a maximum value around $108^{\circ} \mathrm{C}$ and the fast component spectrum disappears around $132^{\circ} \mathrm{C}$. The characteristic temperature at which the fast component spectrum disappears shifts to higher temperatures with increasing content of PEO. These facts are compatible with the UCST type phase diagram for a system comprised of two polymers which have molecular weights of the same magnitude. For instance, the transition temperature of phase separation or molecular mixing has a maximum in the blend ratio of $1: 1$ in the phase diagram of the UCST type.

It is evident that SLPEO chains trapped in the different regions of the phase-separated blend and in the molecular mixed blend have different rates of molecular motion. It can be considered that the size of vacancy in the PEO-rich region is larger than that in the PMMA-rich region or that in the molecular-mixed phase. The mobile fraction estimated from the ESR spectrum is related to the free-volume fraction which is a function of the product of the total number of holes and average hole size. The mobile spin-labels in the holes can rotate isotropically at a rate of approximately $10^{9} \mathrm{~s}^{-1}$ and contribute to the mobile fraction. The structural heterogeneity in local environment of the blend could be clearly observed by the ESR method, indicating change of the total number of holes and/or the average hole size.

\section{Free-Volume Formation and Relaxation in the PEO-Rich Phase of Phase Separated System}

Detection by Spin-Labeled PEO. After the blend is stored at ambient temperature for a long time and phase separated, the sample is aged at temperatures below $23^{\circ} \mathrm{C}$. The sample was stored at a desired temperature for $30 \mathrm{~min}$, heated to room temperature and ESR measurements were carried out at the same temperature. The procedure was repeated with decreasing aging temperatures. Figure 5 shows an example of ESR spectrum observed at room temperature after aging at $-25^{\circ} \mathrm{C}$ for $30 \mathrm{~min}$ (a) in comparison with that before aging (b). Variation in the fractional amount of the fast component spectrum is represented as closed triangles in Figure 3 (A). Surprisingly, the intensity of fast component 


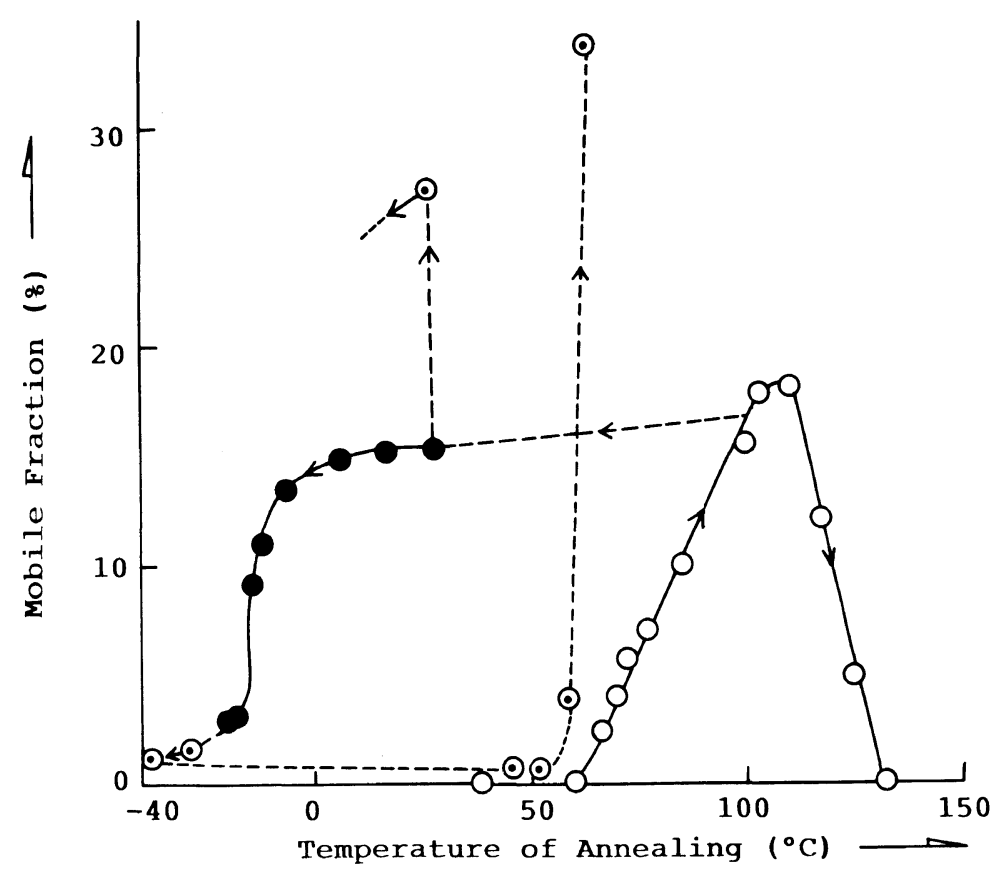

Figure 4. Change in mobile fraction of ESR spectra of spin-labeled PEO in PEO/PMMA (EM1) with annealing temperature after molecular mixing $(O)$ and aging temperature after phase separation $(\Theta, \odot)$. The arrows indicate routes on which changes of the mobile fraction were observed. Measurements were carried out at $23^{\circ} \mathrm{C}$. SLPEO/PMMA $(1: 1)$ : - $\mathrm{O}-$, after molecular mixing; $---\bullet_{---}$and $---\odot_{---}$, after phase separation.

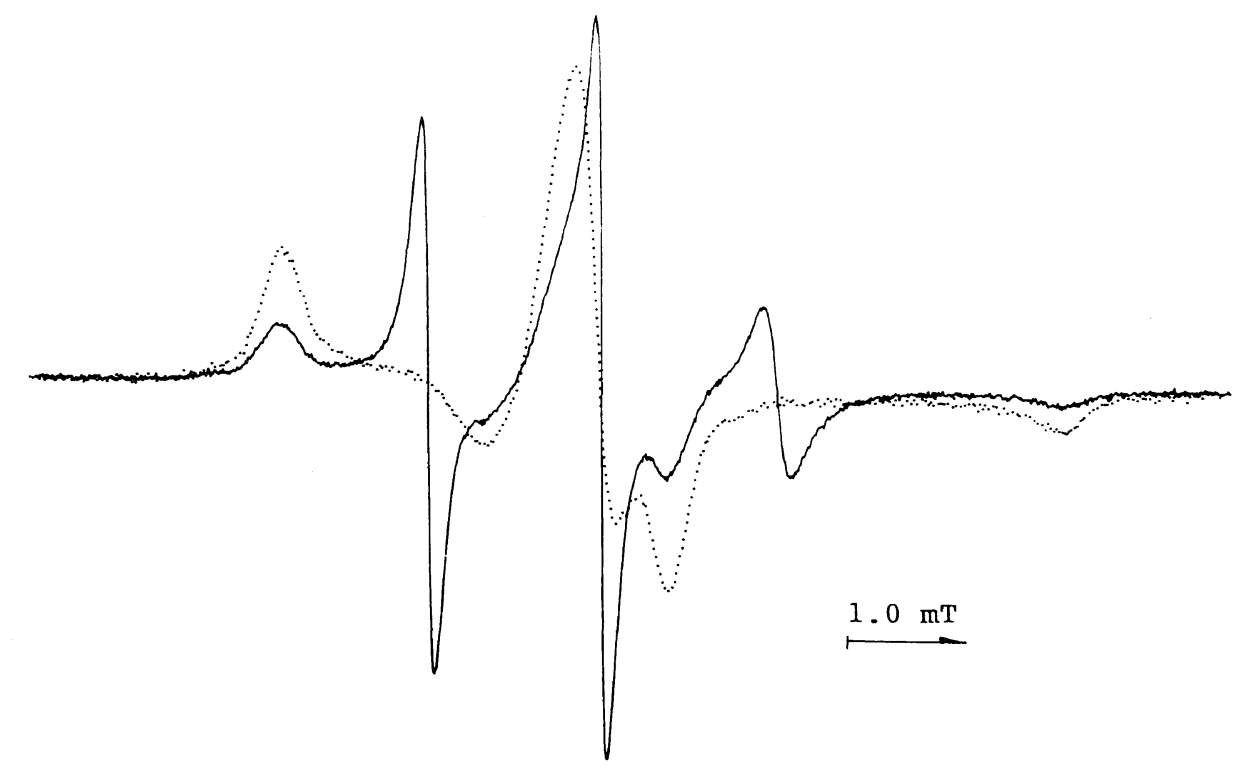

Figure 5. ESR spectra of spin-labeled PEO in PEO/PMMA blend (EM10) after ((a), solid line) and before ((b), dotted line) aging at $-25^{\circ} \mathrm{C}$ for 30 $\min$, (a) - - after aging at $-25^{\circ} \mathrm{C}$ for $30 \mathrm{~min}$; (b) -..--, before aging at $-25^{\circ} \mathrm{C}$ for $30 \mathrm{~min}$.

spectrum decreases with decreasing aging temperature and disappeares finally at $-25^{\circ} \mathrm{C}$. On the other hand, the intensity of the slow component spectrum increases with decreasing aging temperature. The mobile fraction gradually decreases with decreasing aging temperature at $23^{\circ} \mathrm{C}$ to $-10^{\circ} \mathrm{C}$ and drops abruptly around $-13^{\circ} \mathrm{C}$. This reflects that the volume in the PEO-rich phase of the phase-separated system contracts and SLPEO become immobile. For instance, the structural change should involve free-volume relaxation, which was detected by the ESR method.

The PEO/PMMA blend can be considered to have a mobile fraction of more than $28 \%$ in equilibrium state at room temperature. The higher value of mobile fraction after storage at ambient temperature for more than 1 week $\left((\mathbf{A})\right.$ at $23^{\circ} \mathrm{C}$ in Figure 3$)$ than those of the quenched sample $((\triangle)$ in Figure 3$)$ indicates that phase separation occurs at ambient temperature. The phase-separated state after molecular mixing at $142^{\circ} \mathrm{C}$ for $15 \mathrm{~min}$ and annealing at $48^{\circ} \mathrm{C}$ for $30 \mathrm{~min}$ is still non equilibrium because the mobile fraction has a lower value $(20 \%)$ than that after storage at ambient temperature for a long time. The arrows in Figure 3 indicate the route (dotted line) on which changes of the mobile fraction were observed with aging treatments. Volume relaxation occurs in the glassy state and the consequent relaxation proceeds toward 


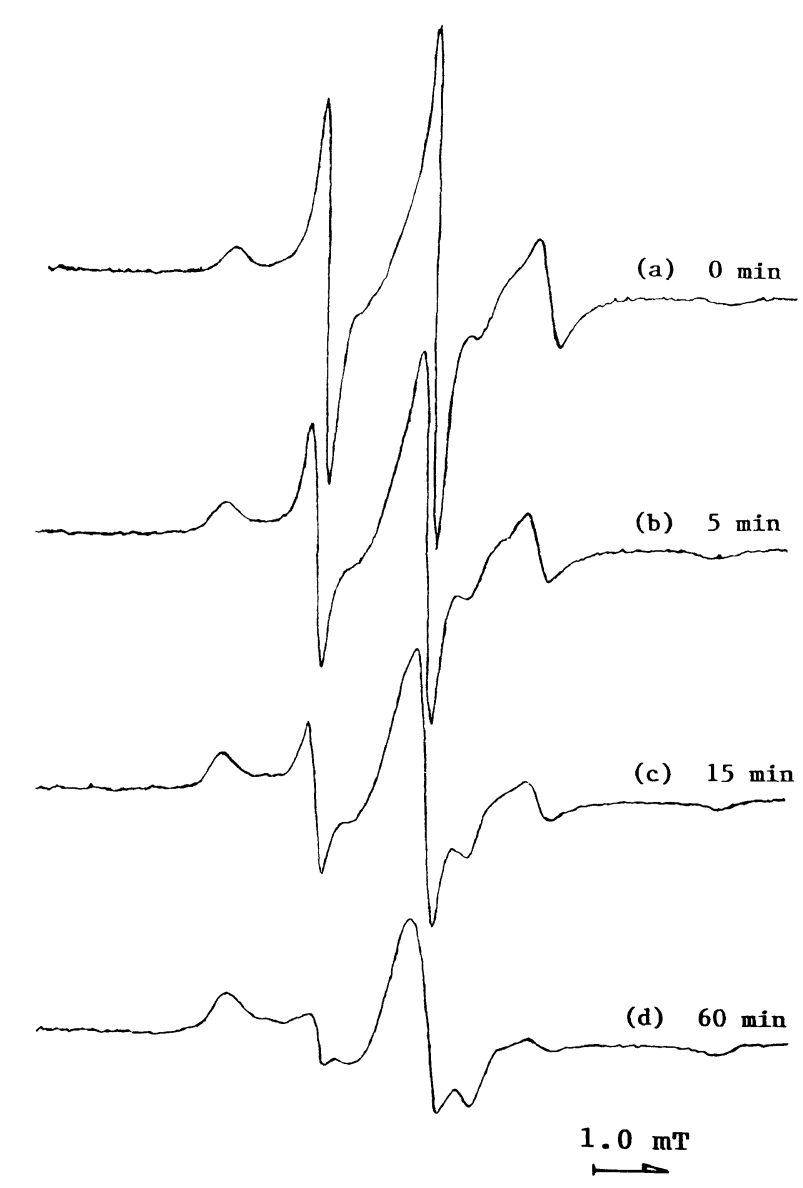

Figure 6. Variation of ESR spectra of spin-labeled PEO in PEO/ PMMA blend (EM4) with aging time at $-13^{\circ} \mathrm{C}$ after phase separation. ESR spectra were observed at $23^{\circ} \mathrm{C}$.

equilibrium. The variation of the ESR spectrum as shown in Figure 5 is similar to that in Figure 1. For instance, the two component spectrum converts to the only one component spectrum by heat-treatments above and below $23^{\circ} \mathrm{C}$. However, the different structural changes, molecular mixing and free-volume relaxation in the PEO-rich phase occur during heat-treatment.

Figure 6 shows variation of ESR spectra of SLPEO in the blend with storage at $-13^{\circ} \mathrm{C}$. The intensity of the fast component spectrum from the mobile radicals decreases with storage time. Thus the amounts and the occupied volumes of mobile SLPEO decrease by volume contraction in the PEO-rich phase. This reflects a relaxation. In Figure 7 , the mobile fraction and its logarithmic are plotted against storage time. Various phenomenological models ${ }^{11}$ of physical aging in glassy polymers have been formulated, which are capable of predicting the time dependent behavior of polymer glasses. The models are based on the concept that deviation of the measurement from equilibrium changes with time according to exponential function as well as non exponential function and multiple rate constants. The results show that the time dependence of the deviation of the fractional free-volume cannot be interpreted in terms of a simple exponential model. At present, we cannot determine what model satisfies our experimental results.

It is worthwhile to note that the structure developed at temperatures below room temperature can be pre-

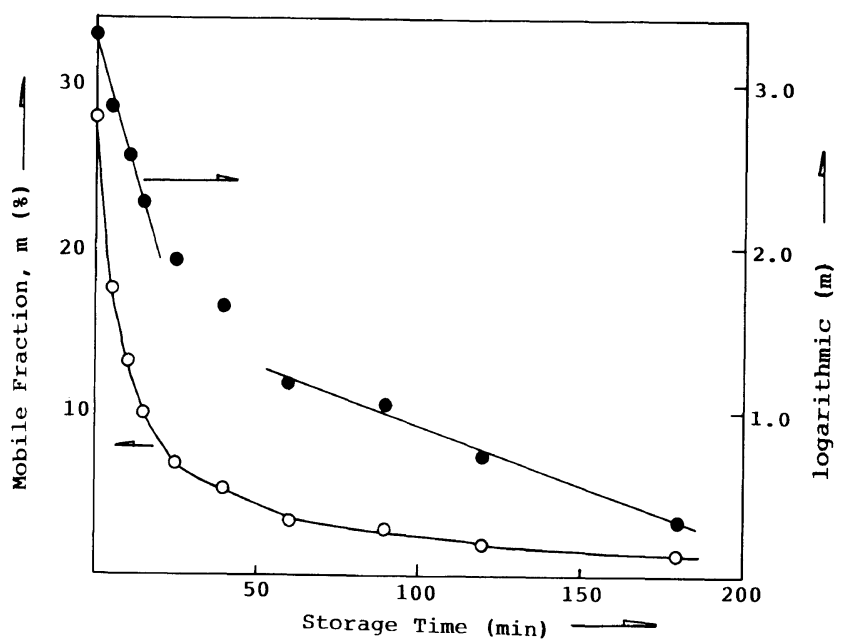

Figure 7. Variation of mobile fraction of ESR spectra of spin-labeled $\mathrm{PEO}$ in PEO/PMMA blend (EM4) with aging time at $-13^{\circ} \mathrm{C}$ after phase separation.

served at the same temperature. The free-volume formation, i.e., volume expansion in the PEO-rich phase cannot be detected within a few hours during storage at room temperature. In other words, the relaxation time for the recovery of the structure is too long to detect by ESR.

After the aging experiments, the sample was annealed at a desired temperature above $23^{\circ} \mathrm{C}$ for $30 \mathrm{~min}$, cooled to room temperature, and ESR measurements were carried out at the same temperature. The closed triangles in Figure $3(\boldsymbol{\Delta})$ show variation of the mobile fraction in the EM10 blend with annealing temperature above $23^{\circ} \mathrm{C}$. The spectrum gave rise to no change during annealing at temperatures below $60^{\circ} \mathrm{C}$ and at last the intensity of the fast component spectrum increased at $60^{\circ} \mathrm{C}$ as shown in Figure 3. Subsequently, the fast component disappeared above $68^{\circ} \mathrm{C}$ because of the molecular mixing of the PEO and PMMA chains.

The same aging experiments for the sample of the EM1 blend were carried out. First, the sample was annealed at $100^{\circ} \mathrm{C}$ for $30 \mathrm{~min}$ and stored at ambient temperature for $c a .1 \mathrm{~h}$, and then the aging experiment was begun. The mobile fraction decreases in the same temperature range (Figure $4(0)$ ) as detected for the EM10 blend. Secondly, the EM1 blend was again annealed at $100^{\circ} \mathrm{C}$ for $30 \mathrm{~min}$; the sample was stored at ambient temperature for 36 days to obtain a completely phase-separated state. The high value of mobile fraction more than $25 \%$ (Figure $4(\odot)$ indicates the phase-separated state. The sample was also annealed after aging for $-40^{\circ} \mathrm{C}$ for $30 \mathrm{~min}$ as shown in Figure $4(\odot)$. A great deal of mobile radicals appeared abruptly at $69^{\circ} \mathrm{C}$ in the EM1 blend. This drastic change in comparison with that in the EM10 blend can be interpreted in terms of the higher transition temperature of the molecular mixing in the EM1 blend. For instance, increasing the mobile fraction due to the free-volume formation, i.e., volume expansion in the PEO-rich phase prevails over decreasing of the fraction due to the molecular mixing.

The free-volume formation causing volume expansion at temperatures higher than $60^{\circ} \mathrm{C}$ was observed by ESR. Experiments on free-volume have involved well-specified 
temperature jumps with subsequent observation of macroscopic quantities such as contraction and expansion of volume. In general, it is difficult to determine what region of a complicated system give rise to change of macroscopic quantities. However, the microscopic quantity, i.e., the mobile fraction estimated by the ESR method is focused on a particular region, the PEO rich

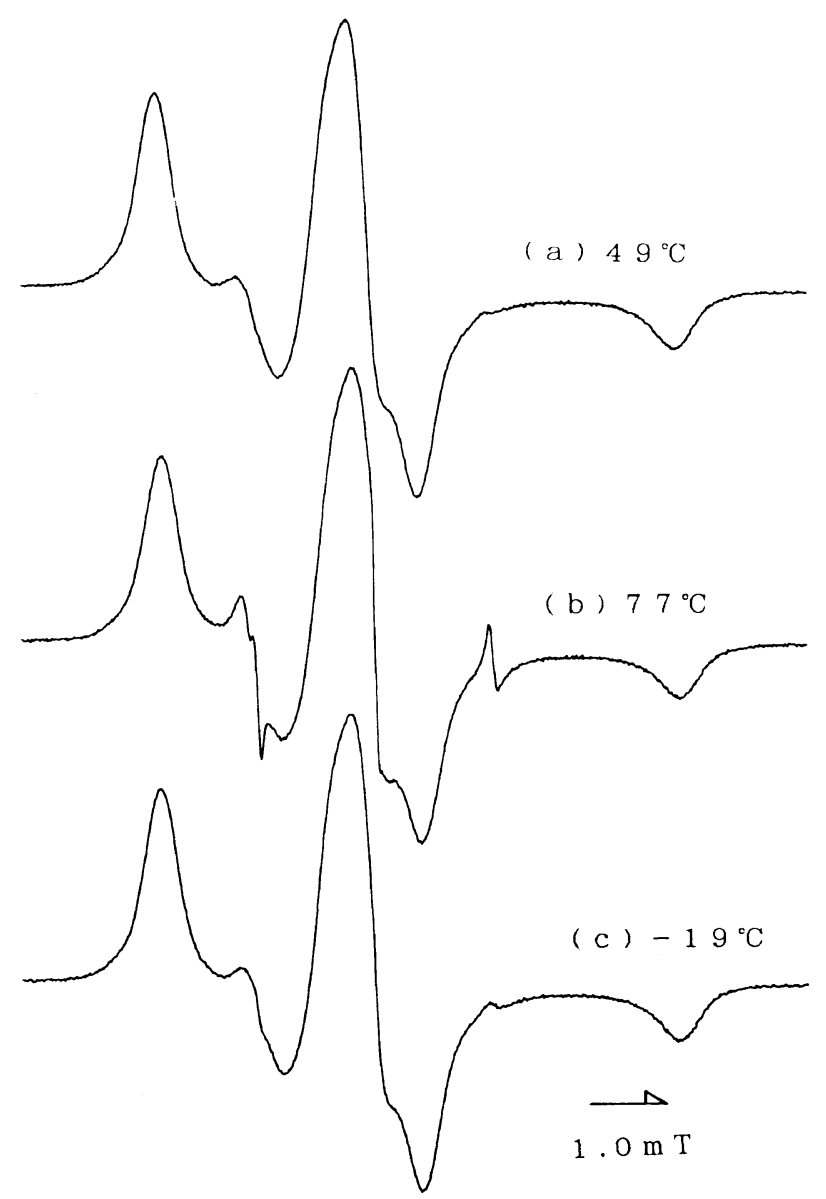

Figure 8. Variation of ESR spectra of spin-labeled PMMA in PEO/PMMA blend (EMM1) with annealing and aging temperature after molecular mixing. ESR spectra were observed at $49^{\circ} \mathrm{C}$. phase in the phase-separated system.

It is very interesting that the spin-label technique is a simple and effective way of studying structural change in a particular region.

Detection by Spin-Labeled PMMA. To elucidate the mechanism of free-volume formation and relaxation in the PEO-rich phase of the phase-separated system, molecular motion of another constituent polymer chains in the PEO-rich phase should be studied. The behavior of SLPMMA was observed in comparison with that of SLPEO. After the EMM1 blend is stored at ambient temperature for a long time (more than 1 week) and phase separated, the sample is aged and annealed at a desired temperature for $30 \mathrm{~min}$, stored to $49^{\circ} \mathrm{C}$, and ESR measurements are carried out at the same temperature. Because the fast component spectrum of SLPMMA was not be observed at room temperature by any heat treatment, all ESR spectra of the spin label were observed at $49^{\circ} \mathrm{C}$ to detect free-volume formation and relaxation. Figure 8 shows variation of the ESR spectra with annealing and aging temperature. Surprisingly, the intensity of the fast component spectrum increases with increasing annealing temperature at $55^{\circ} \mathrm{C}$ to $65^{\circ} \mathrm{C}$ and decreases with aging temperature at $-10^{\circ} \mathrm{C}$ to $-20^{\circ} \mathrm{C}$. The fractional amount of the fast component spectrum is represented as open $(O)$ (annealing) and closed $(O)$ (aging) circles in Figure 9. The temperature ranges of the increasing and decreasing mobile fractiosn coincide with those detected by LSPEO, as shown in Figure 9 (dotted lines). The mobile fractions detected by SLPMMA at high temperature, $49^{\circ} \mathrm{C}$ are smaller than those by LSPEO at low temperature, $23^{\circ} \mathrm{C}$. This indicates that the PMMA chains move more slowly than the PEO chains in the PEO-rich phase and/or that free volume detected by the large LSPMMA is larger than that by small LSPEO in the phase. These experimental facts reflect that the volume in the PEO-rich phase contracts and LSPMMA also becomes immobile. For instance, PMMA chains should also contract and stretch by aging and annealing, respectively, during the same structural changes as detected by LSPEO.

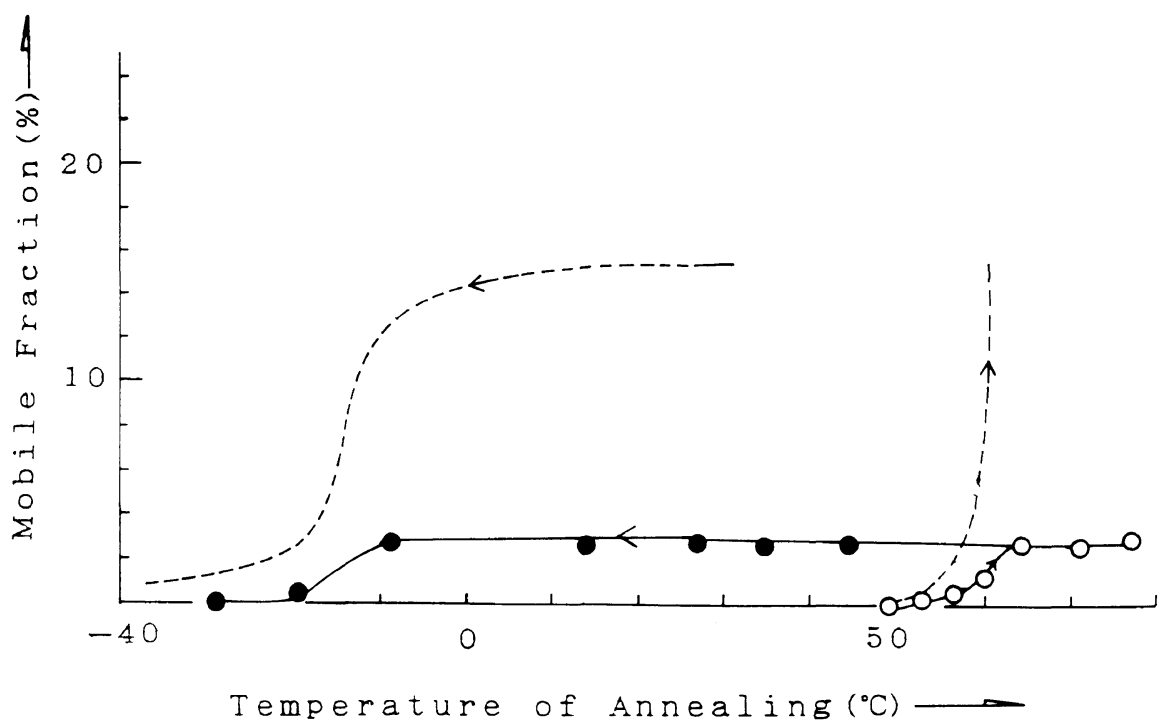

Figure 9. Changes in mobile fraction of ESR spectra of spin-labeled PMMA in PEO/PMMA (EMM1) with aging ( $)$ ) and annealing ( $\bigcirc)$ temperature after phase separation. The arrows indicate the routes on which the changes of the mobile fraction were observed. Measurements were carried out at $49^{\circ} \mathrm{C}$. 


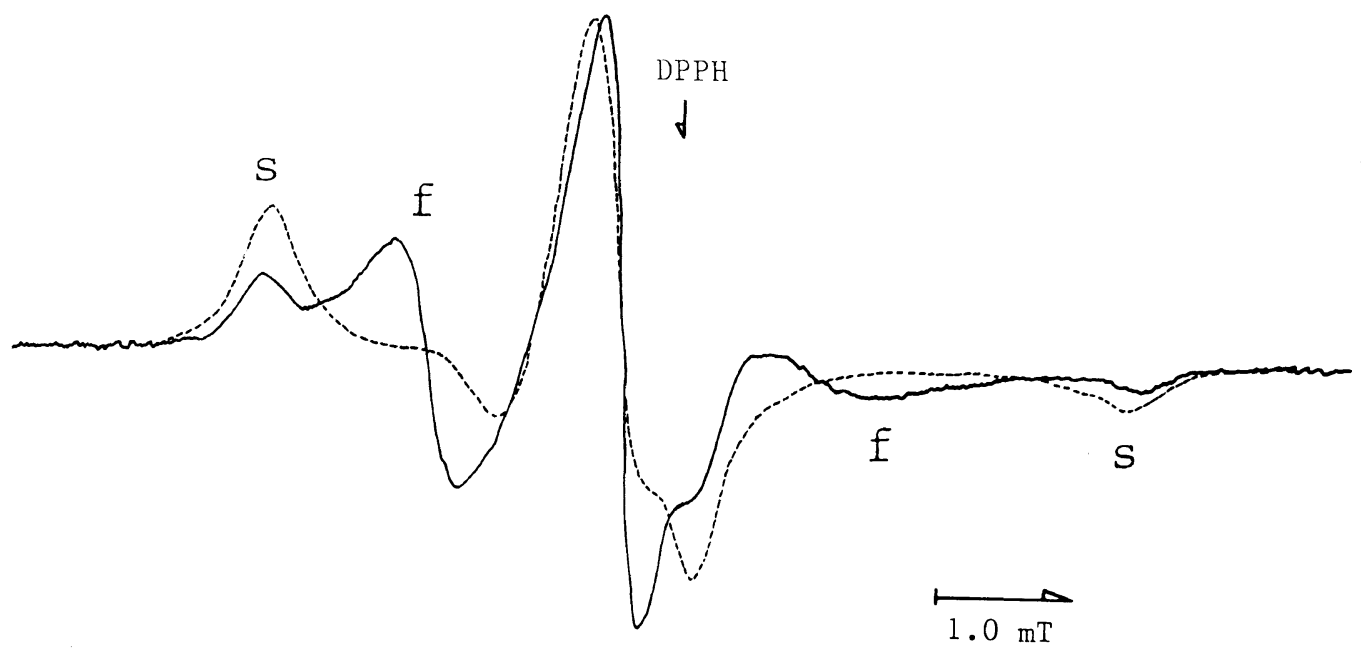

Figure 10. Comparison of ESR spectrum of spin-labeled PEO in phase-separated blend of PEO/PMMA (EM4) ((b), solid line) with that in pure PEO matrices ((a), dotted line). ESR spectra were observed at $4^{\circ} \mathrm{C}$. (a) --..-, SLPEO/PEO; (b) —, SLPEO/PMMA (1:4).

\section{Mechanism of Free-Volume Formation and Relaxation in a PEO/PMMA Blend}

Törmälä et al. $^{7}$ studied solid- and liquid-state relaxation in spin-labeled PEO in detail. We can compare the ESR spectra in pure PEO matrices with those in blends of PEO and PMMA. For the blend, two spectral components with different rates of motion, "fast" and "slow" components arising from the radicals, are observed, whereas only one component is detected over the entire temperature range for pure matrices. The fast and slow components observed in the phase-separated blend can be attributed to radicals in the PEO-rich and PMMA-rich phases, respectively. Figure 10 shows the ESR spectrum of SLPEO in the phase-separated blend (b) in comparison with that in pure PEO matrices (a). The ESR spectra were observed at $4^{\circ} \mathrm{C}$ between room temperature and $-13^{\circ} \mathrm{C}$ after the samples were stored at ambient temperature for a long time (more than 1 week). The line width of the fast component spectrum in the blend broadens because of motional slowing in comparison with that shown in Figure 1a. On the other hand, the fast component spectrum is not observed in pure PEO matrices. This is inconsistent with the glassrubber transition temperature of PEO $\left(-65^{\circ} \mathrm{C}\right)$ lower than that of isotactic PMMA $\left(60^{\circ} \mathrm{C}\right) .^{4 \mathrm{~b}}$ The closer location of even small amounts of PMMA chains should cause hindrance of the mobility of the PEO chains.

How can we interpret this discrepancy? In phase separation, PEO-rich and PMMA-rich phases appear by translational molecular diffusion of constituent polymer chains. PMMA chains are excluded gradually and equilibrium concentration of PMMA chains can be attained in the PEO-rich phase. A lot of vacancies may be generated during the phase separation if the molecular mobility of the PEO and PMMA chains is much different. The more rigid PMMA chains prevent vacancies from disappearance. In other words, the PMMA chains support the vacancies as posts. PEO chains trapped in vacancies have high molecular mobility and the size and number of vacancies should be related to the fractional free-volume as a function of the fractional amount of the fast component spectrum. PMMA chains trapped in

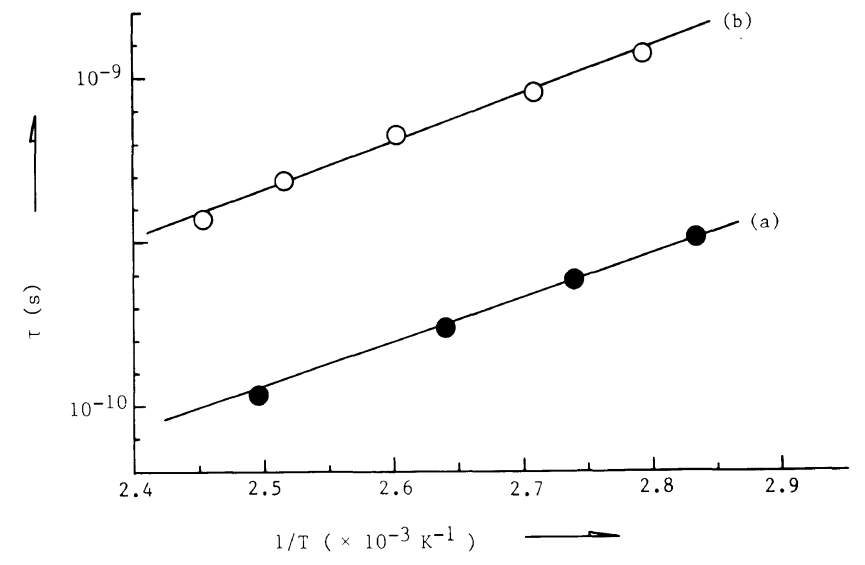

Figure 11. Comparison of temperature dependence of rotational correlation time $(\tau)$ of spin labeled PEO (a) with that of spin-labeled PMMA (b) in the same matrices of the PEO/PMMA blend (EM1 or EMM1). (a), SLPEO/PMMA (1:1); (b), SLPMMA/PEO (1:1).

the vacancies of the PEO-rich phase have high molecular mobility as mentioned in the previous section. The rate of molecular motion of the chains in the blend can be considered to be lower than that of PEO chains because of the bulky side chains of PMMA. To confirm differences in mobility of PEO and PMMA chains, the rotational correlation times of SLPEO are compared with those of SLPMMA in the same matrices of the blend. Figure 11 shows the temperature dependence of the rotational correlation times in the higher temperature range, obtained from the temperature dependence of ESR spectra of radicals using Kivelson's theory on the assumption of isotropic molecular motion. ${ }^{12}$

$$
\tau=15 \pi \sqrt{3} W_{0}\left\{\left(h_{0} / h_{1}\right)^{0.5}-\left(h_{0} / h_{-1}\right)^{0.5}\right\} /(8 b \Delta \gamma H)
$$

Here, $h_{1}, h_{0}$, and $h_{-1}$ are respective peak heights of three components, $W_{0}$ is the line width of central component, $H$ the static magnetic field applied, and $b$ and $\Delta \gamma$ the quantities for the anisotropic of $g$ - and $A$-tensors. PEO and PMMA chains are molecularly and uniformly mixed with each other at the molecular level in the temperature range. 
The rates of the molecular motion of the LSPEO are ca. 5 times higher than those of SLPMMA in the same matrices of the PEO/PMMA $(1: 1)$ blend.

After phase separation, ESR spectra of both labels are observed at room temperature. Two spectral components with different rates of motion arising from SLPEO are observed, whereas only one component with slow rate of motion, arising from SLPMMA is detected. This suggests that PEO chains are more mobile than PMMA chains in the PEO-rich phase of the phase separated system at room temperature.

In general, segmental motion of polymer chains stops and polymer chains contract at glass-rubber transition temperature $\left(T_{\mathrm{g}}\right)$ with cooling. In other words, freevolume relaxation occurs. In PEO/PMMA blends, the more immobile PMMA chains contract at first and PEO chains are still mobile near $T_{\mathrm{g}}$ because of the mobility of constituent polymer chains. Structural changes next proceed toward glassy and equilibrium state and finally PEO chains become immobile.

When the sample is heated to room temperature after aging, the state of volume contraction seems preserved for a few hours. PMMA chains cannot stretch instantly because of the rigid structure and SLPEO is still immobile. PMMA chains begin to stretch at $60^{\circ} \mathrm{C}$ near $T_{\mathrm{g}}$ of isotactic PMMA and free volume is generated. These relaxation phenomena can be considered to be reflected on the experimental results mentioned in the previous section.
Acknowledgments. The author is grateful to Dr. Hori (Nagoya Institute of Technology) and Mr. Kashima (Teijin Chemical Ltd.) for helpful discussion and preparation of samples.

\section{REFERENCES}

1. For example: L. A. Utracki, "Polymer Alloys and Blends," Hanser, New York, N.Y., 1989.

2. For example: G. Greiner and F. R. Schwerzl, Colloid Polym. Sci., 267, 39 (1989).

3. For Example: G. Brinke and R. Grooten, Colloid Polym. Sci., 267, 992(1989).

4. Some authors have studied blends of PEO and PMMA, for example: (a) X. Li and L. Hsu, J. Polym. Sci., Polym. Phys. Ed., 22, 1331 (1984). (b) C. Silverstre, S. Cimmino, E. Martuscelli, F. E. Karasz, and W. L. Macknight, Polymer, 28, 1190 (1987). (c) E. John, and T. Ree, J. Polym. Sci., Polym. Chem. Ed., 28, 385 (1990). (d) C. Marco, J. G. Fatou, M. A. Gomez, H. Tanaka, and A. E. Tonnelli, Macromolecules, 23, 183 (1990).

5. S. Shimada, Y. Hori, and H. Kashiwabara, Macromolecules, 21, 979 (1988), and references therein.

6. S. Shimada, Y. Hori, and H. Kashiwabara, Macromolecules, 23, 3769 (1990)

7. P. Törmälä and L. Lindlberg, Polymer, 14, 481 (1973).

8. K. Hatada, K. Ute, K. Tanaka, T. Kitayama, and Y. Okamoto, Polym. J., 17, 997 (1985)

9. T. Kurosaki, K. Lee, and M. Okawara, J. Polym. Sci., Polym. Chem. Ed., 10, 3295 (1972).

10. S. Shimada, Y. Hori, and H. Kashiwabara, Macromolecules, 25, 2771 (1992).

11. J. G. Victor and J. M. Torkelson, Macromolecules, 21, 3490 (1988), and references therin.

12. D. Kivelson, J. Chem. Phys., 33, 1107 (1960). 